\title{
Perfil dos Pacientes Submetidos à Intervenção \\ Coronariana Percutânea no Serviço de Hemodinâmica do Hospital Universitário Sul Fluminense, Vassouras - RJ
}

\author{
Alan Marcelo Oliveira São Leão \\ Discente do curso de Medicina, Universidade Severino Sombra \\ allan2lufba@hotmail.com \\ Marlon Mohammed Vilagra \\ Docente do curso de Medicina, Universidade Severino Sombra \\ marlon.vilagra@uol.com.br
}

\begin{abstract}
Resumo: a intervenção coronariana percutânea (ICP) é um método rápido, pouco invasivo, seguro e muito eficaz que tem sido cada vez mais utilizado no diagnóstico e no tratamento da doença arterial coronariana (DAC) nos centros de atendimento cardiovasculares emergenciais e urgenciais. Métodos: selecionados 157 pacientes, submetidos à intervenção coronariana percutânea, em um período de seis meses entre 2009 e 2010, para traçar um perfil epidemiológico e fisiopatológico das obstruções por DAC. Resultados: os pacientes que realizaram ICP eram significativamente mais velhos, em sua maioria eram homens, mas que apresentavam menor porcentual de doença coronariana multiarterial. Conclusão: neste registro municipal, a artéria coronária descendente anterior (DA) foi a mais acometida pela obstrução.
\end{abstract}

Palavras-chaves: Intervenção coronariana percutânea. Doença arterial coronariana. Hemodinâmica. Angiografia cardíaca.

\section{Profile of Patients Undergoing Percutaneous Coronary Intervention in the Hemodynamics Service of University Hospital South Fluminense, Vassouras - RJ}

\begin{abstract}
Percutaneous coronary intervention (PCI) is a rapid, minimally invasive, safe and very effective method. It has been increasing widely used in the diagnosis and treatment of coronary artery disease (CAD) in emergency cardiovascular care centers and urgencies. Methods: We selected 157 patients undergoing percutaneous coronary intervention in a period of six months between 2009 and 2010, drawing an epidemiological and pathophysiological obstructions by CAD. Results: Patients who underwent PCI were significantly older, were mostly men, but had a lower percentage of multivessel coronary artery disease. Conclusion: In this city record, the anterior descending coronary artery (DA) was the most affected by the obstruction.
\end{abstract}


Keywords: Percutaneous coronary intervention. Coronary artery disease. hemodynamics. Cardiac angiography.

\section{Introdução}

As doenças cardiovasculares (DCV) no início do século $\mathrm{XX}$ eram responsáveis por menos de $10 \%$ dos óbitos em todo o mundo, mas ao final desse mesmo século, esse grupo de doenças foi o responsável por, aproximadamente, $50 \%$ dos óbitos nos países desenvolvidos e $25 \%$ naqueles em desenvolvimento. São estimados 25 milhões de óbitos em 2020, sendo que a maior causa de morte serão as doenças isquêmicas do coração, superando os casos de doenças infecciosas. (Almeida et al., 2003).

A insuficiência cardíaca e suas complicações hoje são reconhecidas como um problema crescente e importante de saúde pública. Fatores como a industrialização e a urbanização implicaram mudanças na dieta alimentar, aumento do tabagismo, sedentarismo e obesidade. A consequência natural desse processo é o desenvolvimento da hipertensão arterial, diabetes e doença das artérias coronárias, sendo a insuficiência cardíaca a via final dessas e de outras doenças. Estima-se que 6,4 milhões de brasileiros sofram de insuficiência cardíaca. (Neto et al., 2004).

Nas últimas duas décadas ocorreram grandes avanços no reconhecimento e no manejo de pacientes com síndrome coronária aguda (SCA), com ou sem supradesnivelamento do segmento de ST, e na implementação de regimes antitrombóticos e antiplaquetários potentes associados a algoritmos de estratificação de risco o que determinou significativa redução das taxas de óbito, infarto e isquemia recorrente. A coronariografia e a intervenção coronária percutânea (ICP) adquirem importância fundamental nesse cenário. (Santos et al., 2009).

As síndromes coronárias agudas (SCA) também chamadas de doença arterial coronariana (DAC) englobam um grupo de entidades que incluem infarto agudo do miocárdio (IAM) com supradesnível do seguimento ST e angina instável. Manifestações como essas são causas comuns de atendimento e de admissões nos departamentos de emergência, assim como representam a principal causa de morbidade e mortalidade cardiovasculares em todo o mundo. Segundo dados epidemiológicos americanos foi relatado que mais de 12 milhões de pessoas têm a doença arterial coronariana e ainda, mais de um milhão de pessoas experimentam um infarto de miocárdio anualmente, o que resulta cerca de 466 mil mortes atribuídas à DAC. No Brasil, $32,6 \%$ dos óbitos com causa confirmada estão relacionados às doenças cardiovasculares. (Almeida et al., 2003).

Existem diversos fatores de risco associados à DVC e que também podem contribuir para o surgimento de doença arterial coronariana. Entre eles podemos citar: idade avançada, sexo masculino, hipertensão arterial sistêmica, dislipidemia, esta representada por níveis séricos elevados das lipoproteínas de baixa densidade (LDL-colesterol) e por níveis séricos baixos das lipoproteínas de alta densidade (HDL-colesterol), seguidos de histórico familiar para DAC, diabetes melito e tabagismo. (Armaganijan et al., 2000). O risco de doença cardiovascular em fumantes é de duas a quatro vezes maior. A hipertensão arterial é um dos fatores de risco fundamental para o desenvolvimento de doenças cardiovasculares. Portanto, populações de diferentes faixas etárias podem necessitar de uma intervenção 
cardíaca, em especial a ICP, dependendo da patologia que venha apresentar, o que pode ou não ser acompanhado de doenças pré-existentes.

O diabetes melito é uma doença crônica frequente para a qual estima-se um aumento de prevalência em aproximadamente $30 \%$ até o final da próxima década, segundo a Organização Mundial de Saúde (OMS). No Brasil, estima-se, existem 5 milhões de diabéticos. Na faixa etária de 60 a 69 anos a prevalência do diabetes melito é de 17,4\%. (Lerario et al., 1998).

O cateterismo cardíaco e a angiografia cardíaca aliados aos procedimentos modernos, seguros e efetivos de cardiologia intervencionista (não cirúrgica) oferecem uma avaliação detalhada da anatomia e fisiologia da vasculatura e do coração. Em um contexto em que haja uma ou mais lesões anatomicamente abordáveis e responsáveis por isquemia clínica ou para as quais a intervenção coronariana percutânea (ICP) constitui a abordagem preferida pelo paciente que compreende os riscos e benefícios das alternativas terapêuticas, esses métodos diagnósticos e terapêuticos constituem o padrão ouro na avaliação e no tratamento das cardiopatias. A ICP é responsável pela maioria das revascularizações miocárdicas e representa, atualmente, a forma mais comum desse procedimento.

A ICP, também chamada de angioplastia coronária transluminal percutânea (ACTP), é uma moderna modalidade terapêutica extremamente útil no tratamento de lesões coronarianas oclusivas e/ou semioclusivas, a chamada doença arterial coronariana (DAC), além de permitir a abordagem de lesões localizadas na porção distal de vasos, lesões difusas e calcificadas de múltiplas artérias coronárias ou enxertos de veias safenas ou mamárias anteriormente implantados. Estudos comparativos que envolvem ICP demonstraram que as taxas de mortalidade eram semelhantes em lesões multivasculares, comparados os pacientes tratados com revascularização miocárdica (exceto aqueles com diabetes, nos quais a taxa de mortalidade foi menor do que naqueles com a ICP).

O pós-procedimento da ICP cardíaca apesar de ser um procedimento não invasivo e rápido se caracteriza por um período crítico e delicado, com a possibilidade de surgimento de complicações, das quais podemos citar as pulmonares, digestivas, neurológicas, renais, cardíacas e infecciosas.

O Serviço de Hemodinâmica do Hospital Universitário Sul Fluminense que atende a uma parcela considerável da população que mora no estado do Rio de Janeiro, é considerado uma das referências estaduais na prestação desse tipo de procedimento, pois tem um corpo clínico formado por médicos cardiologistas, cirurgiões cardíacos e cardiologistas intervencionistas, além de equipes de Fisioterapia, Enfermagem, Nutrição e Psicologia.

Portanto, se faz necessária a realização de um trabalho que investigue o perfil da população neste hospital atendida para que se compreendam os fatores que interferem em sua evolução com o sentido de melhorar a assistência prestada por parte das equipes.

\section{Objetivo}

O presente estudo tem como objetivo determinar o perfil dos pacientes submetidos à ICP atendidos no Serviço de Hemodinâmica do Hospital Universitário Sul Fluminense (HUSF) 
em Vassouras - RJ, assim como quantificar o número de lesões arteriais e identificar as principais artérias acometidas.

\section{Materiais e Métodos}

Foi realizado um estudo retrospectivo, de natureza documental, com base na análise dos laudos e prontuários que envolvem 157 pacientes submetidos à ICP no Instituto Sul Fluminense de Cardiologia, no período que compreende os meses de novembro de 2009 e abril de 2010. A doença arterial coronária (DAC) significativa foi considerada com a confirmação da obstrução de mais de $50 \%$ da luz arterial.

\section{Resultados}

Foram analisados 157 pacientes que se submeteram à ICP, dos quais 147 (94\%) apresentaram angioplastias eletivas e outras 10 (6\%), primárias. Destes, 102 (65\%), eram do sexo masculino e 55 (35\%), do feminino. Em relação ao número de lesões, 79 (50\%), apresentaram lesão uniarterial, 41 (26\%), biarterial e outros 37 (24\%), multiarterial. A Artéria DescendenteAnterior(DA) foi lesionada em $43 \%$ dos casos, seguida pela Coronária Direita (CD) em 28\%, Circunflexa (CX) 22\% e a Diagonalis (DG) em 7\%. Apenas duas angioplastias não obtiveram sucesso em sua realização, o que torna necessária abordagem cirúrgica sem intercorrência.

\section{Discussão}

O estudo de Framingham demonstra que a incidência da DAC é menor nas mulheres tanto na pré como após a menopausa, além de existir um intervalo de sete a dez anos de proteção na incidência da DAC nas mulheres (Kannel et al., 1976). Admite-se que esta diferença decorra do estado hormonal, pois se sabe que a incidência da DAC é menor na pré-menopausa, provavelmente devido ao efeito protetor do estrogênio, o que aumenta significativamente após a menopausa. Os efeitos protetores do estrogênio estão relacionados com o melhor perfil lipídico, metabolismo da glicose, menor fibrinogênio sérico e com a ação direta do estrogênio no sistema vascular, com inibição da aterogênese e a trombogênese. Após a menopausa, com a redução das concentrações séricas de HDLcolesterol e com o aumento de LDL-colesterol instalar-se-ia um perfil lipoproteico de risco para a DAC (Mansur et al., 2000). Neste estudo, foi possível observar uma menor incidência de DAC nas mulheres.

O exponencial crescimento da prática da intervenção coronariana percutânea no Brasil está documentado por meio do registro CENIC (Central Nacional de Intervenções

Cardiovasculares), órgão oficial da SBHCI (Sociedade Brasileira de Hemodinâmica e Cardiologia Intervencionista) que, desde 1991, contabiliza os procedimentos percutâneos cardiovasculares realizados em nosso país. A supremacia dos stents coronarianos fica bem caracterizada e é possível observar uma melhoria nos resultados imediatos, com maior sucesso e redução nas complicações (Sousa et al., 1998). 
A indicação de intervenção coronariana percutânea ocorreu em 65,4\% dos pacientes com IAM com SST e em 25,6\% dos pacientes com IAM sem SST, consideravelmente mais elevada do que a relatada no estudo ENACT (83\%) (Santos et al., 2006).

A Intervenção Coronária Percutânea apresenta uma série de vantagens quando comparada à cirurgia cardíaca. Dentre elas é importante ressaltar o menor risco de morbi-mortalidade cerebrovascular, pulmonar, digestiva e renal, principalmente em pacientes idosos e em portadores de doença prévia destes sistemas, além do tempo mais curto de convalescença e de internação hospitalar; do retorno às atividades profissionais e até de menores custos hospitalares, após a alta do paciente (Zago et al., 2002).

Ao realizar comparativos com dados do Global Registry of Acute Coronary Events (GRACE), aproximadamente, mais da metade dos pacientes apresentava uma idade superior a 65 anos. (Avezum et al., 2004); forma semelhante aos pacientes admitidos no Serviço de Hemodinâmica do Hospital Sul Fluminense, com predominância na faixa etária dos 60 aos 69 anos. O maior índice de indivíduos nesta faixa etária está relacionada com o fato de que com o passar dos anos, o sistema cardiovascular sofre uma série de alterações como arteriosclerose, diminuição da distensibilidade da aorta e das grandes artérias, comprometimento da condução cardíaca e redução na função barorreceptora. Além da idade, há outros fatores de risco como hipertensão, diabetes mellitus, fumo, dislipidemias, sedentarismo e obesidade (Porto et al., 1998).

Neste estudo o número de vasos acometidos em pacientes com doença arterial coronariana (DAC) foi de $50 \%$ de lesões uniarteriais, $26 \%$ de lesões biarteriais e os outros $24 \%$ de lesões multiarteriais. Isso é explicado pela própria história natural da fisiopatologia da DAC. O mais comum é que haja primeiro uma lesão uniarterial e, posteriormente, se desenvolvam lesões em outras coronárias, de acordo com a evolução da DAC. (Zago et al., 2002).

É importante mencionar que a identificação das lesões em uniarterial, biarterial ou multiarterial está diretamente relacionada com o tratamento do paciente. Desse modo, é sabido que a intervenção coronariana percutânea de estenoses em bifurcação permanece um desafio. Os resultados observados com o balão (kissingballoon) são pobres. As técnicas atero-ablativas de execução complexa e o implante de stents coronarianos de primeira geração também ofereceram dificuldades técnicas. Além disso, seis trabalhos multicêntricos e randomizados compararam, em pacientes com doença multiarterial, os resultados da angioplastia com o balão e os da cirurgia de revascularização e demonstraram que ambas as possibilidades de tratamento exibiam resultados tardios similares em termos de sobrevida e taxas de infarto do miocárdio (Diretriz de indicações e utilizações das intervenções percutâneas e stent intracoronariano na prática clínica, 2003).

\section{Conclusão}

Houve prevalência de pacientes do sexo masculino, em sua maioria alocada na faixa etária entre 60 e 69 anos. A maioria dos procedimentos realizados no serviço foram de ICPs eletivas. O tipo de lesão mais comum foi uniarterial. A artéria mais abordada foi a DA, sendo a via radial a mais utilizada para o procedimento. 


\section{Referências}

Almeida FF, Barreto SM, Couto BRGM, Starling CEF. (2003). Fatores Preditores da Mortalidade Hospitalar e de Complicações Pre Operatórias Graves em Cirurgia de Revacularização do Miocárdio. Arquivo Brasileiro de Cardiologia 2003; 80: 41-50.

Armaganijan D, Batlouni M. (2000). Impacto dos Fatores de Risco Tradicionais. (Revista da Sociedade de Cardiologia do Estado de São Paulo 2000, nov/dez; 10(6): 686693).

Avezum, Álvaro et. al. (2004). III Diretriz sobre tratamento de infarto agudo do miocárdio. Arq. Bras. Cardio. $12004 ; 83$ : 1-86.

Kannel WB, Hjortland MC, McNamara PM, Gordon T. (1976) Menopause and risk of cardiovascular disease: The Framingham Study. Ann Intern Méd 1976;85:447-52.

Lerario AC. Diabete Melito: Aspectos Epidemiológicos. (1998) Revista da Sociedade de Cardiologia do Estado de São Paulo 1998, set/out; 8(5): 885-89.

Mansur AP, Aldrighi JM. Doença coronária na mulher. (2000) Rev Bras Méd 2000; 57:440-5.

Mattos LA, Sousa AGMR, Pinto IMF, Silva ER, Carneiro JK, Sousa JE, Mangione J. A, Caramori P, Lima V, Bueno RL. (2004). Uma Comparação Entre a Intervenção Coronariana Percutânea de Resgate e Primária Realizadas no Infarto Agudo do Miocárdio. Relato Multicêntrico de 9.371 Pacientes. Arquivos Brasileiros de Cardiologia. 82 (5): 434-43.

Neto JMR. (2004). A Dimensão do Problema da Insuficiência Cardíaca do Brasil e do Mundo. Revista da Sociedade de Cardiologia do Estado de São Paulo 2004, jan/fev; 14(1): 1-10.

Porto e cols. (1998). Doenças do Coração: Prevenção e Tratamento. Guanabara Koogan, 1998.

Santos EL, Minuzzo L, Pereira MP, Castillo MTC, Palácio MAG, Ramos RF, Timerman, Piegas LS (2006). Registro de Síndrome Coronariana Aguda em um Centro de Emergências em Cardiologia. 87 (5).

Santos ES. Cardiologia no Pronto Socorro. (2009). Revista da Sociedade de Cardiologia do Estado de São Paulo 2009, abril/maio; 19: 94-147).

Sousa AGMR, Mattos LA, Moura Campos Neto C, et al. (1998). Intervenções percutâneas para revascularização do miocárdio no Brasil em 1996 e 1997 comparadas às do biênio 1992-93. Relatório do registro CENIC (Central Nacional de Intervenções Cardiovasculares). Arq Bras Cardiol 1998; 70:423-30.

Zago e cols. Evolução para Tratamento da Doença Coronária Multiarterial. (2002) Arq. Bras. Cardiol. 2002; 78: 250-60). 